\title{
Modern Teaching of Art and Design Indoor and Outdoor Manuscripts
}

\author{
Zhihua Xu \\ College of Art Design, Jingdezhen Ceramic Institute, Jingdezhen, China
}

Keywords: art and design indoor, outdoor, painted, computer

\begin{abstract}
In the twenty-first century now, people are accepting the rapid development of the information age, while emphasizing the fast-paced, high efficiency. And we are also the times of higher education, popularization and application of multimedia teaching. So that educators and students are in conflict with traditional teaching and learning methods, and as a special sector of our art and design education, the use of computers is indispensable , and is a very important one to learn and use tools, But the attendant computer renderings can be said to have replaced the traditional hand-painted renderings status in the past, then the design of the manuscript as well as its position ? The answer is yes, fifteen years ago , I purchased a computer began to study Adobe Photoshop CS. And teaching about the software (Corduroy, AutoCAD) so far. And I have always believed in the program design phase, design manuscripts as the behavior of the human brain design thinking process is computer graphics can not be replaced.
\end{abstract}

\section{Introduction}

Today's students, their brain, eyes, ears all the time to receive a new message , from the use of mobile phones, QQ, MP4, computer access, etc. , I can see their hearts are restlessness, and these high-tech products has become indispensable part of their lives . In addition to classes, most of the time is in the Internet, coexistence between man and computer, and even shopping are on the Internet, online dating, nor is it not strange to see things fresh.

Art and design students of classwork one hand, waiting is back home to open the computer, check the information, and even some students on the Internet borrowed other people's works directly as existing, outrageous works as existing after the later of the door Lesson almost not come on , in order to complete the job tasks. Seems to be the era of computer networks to bring them all good luck and high returns carrier, very few people are willing to hand to outline drawn manuscript , and performed the original design thinking, and seems to have been designed in saying manuscript obsolete. Because the field of computer graphics design education colleges in popularity, design manuscript was left out even relatively neglected, but the long-term development to master the traditional performance techniques - hand-painted manuscript, rests in the preliminary stages of the design of classroom teaching and university staff today remains particularly important.

\section{The definition of art and design of the manuscripT}

Art and Design is a graphic design industry manuscript language , is an expression of intent and concept design industry with the images in this visual form ; are necessary to reflect, exchange, transfer art design concept symbol vector behavior has free and easy, casual generalization , university Quick, concise performance advantages. Art and design hand-painted manuscripts and many more.

\section{Draw the design of the manuscript}

Design manuscripts performance is good or bad, as long as the design intent from manuscript to read . No matter what the drawing tools to draw bold. As long as good at seizing every opportunity to practice freehand, with the long and arduous effort, painting skills will become increasingly skilled. 


\section{Process weapon - Design Manuscript}

Tools designed to facilitate the application of the manuscript should be used , and easy to carry. Factorial design manuscript to have clear reasons for drawing, pen, disposable pens than a pencil or other drawing tools easier to use. I believe that the pen more appropriate. Manuscript itself should be happy the product creation process, but does not require it to become an exhaustive 's complete works. Allow students to feel at liberty to freely draw, and sketched out a variety of ideas. So that the original idea to record keeping, and free to feedback ; and easy to put together various images of the manuscript to compare, thereby achieving further deepening of the design.

Manuscript stage design students need to undertake the following tasks: first to collect a variety of data and information and design issues related to ( the site requirements, customs ) ; second to analyze these data and information to gain an understanding of the design issues ( relationship , the level of need ) ; and the third to propose solutions to problems ( narrative , programs manuscript ) . Fourth, we should draw the floorplan layout should be based on rational, scientific , focusing on the functional design of the platform.

\section{Point perspective based, isometric drawings , supplemented}

In the interior design is the easiest to draw a little perspective, is the most practical perspective , it can be directly used to represent isometric drawings, isometric drawing techniques similar cavalier perspective of Chinese painting , the viewer is not from one point to observe the scene , and exposure to the front of all the scene, the viewpoint is changing. Advantages isometric view is that both the performance of the three-dimensional space while maintaining a cross-sectional plane and the "true" scale . Relies heavily on the performance of the design ; This means strengthening the manuscript thinking thinking rich performance and rich performance. Painting legalistic only lead to legalistic thinking. In addition, some available with arrows lead to labeling materials, construction requirements.

\section{Art design features manuscripts}

Former member of the Academic Committee of the State of Art , the Art Institute of Suzhou University tenured professor Zhang Road, Sirs, Suzhou University College of the Arts Kai Zhuge often say : "Good memory as bad written ; the computer is only a tool not a substitute for thinking ." In the classroom often say these Bo , Master , you have to do notes, multi- sketched design manuscript. And we must strengthen this aspect of your student's basic training . That is, to carry a sketchbook, ready to record your visual stimulation genus intent, if not with a sketchbook , you can call the school in the future, failure to criticize the teaching reform, but we can not call me ( kai Zhuge ). Mr. seen two manuscripts of sketches of how attention. At the graduate level of study, our students and many of them are the backbone of the professional colleges of art and design teachers, when we are talking about gossip design thinking design manuscripts, Many of the students are computer experts , they agreed that the sketch design ideas upon which inherited the manuscript is an important means of design , computer renderings present insurmountable highly personalized visual symbols media. Enrollment and network information age college students to design manuscript led to decline in the quality are inseparable.

Historically, many creative designers, architects have excellent freehand drawing skills, such as advocating less is more Mies van de Luo, Wright, IM Pei , Meier , Peter Eisenman , Fallon left Piano , Rogers , Tadao Ando , Toyo Ito , Steven hall , Norman Foster , Frank Gehry , Hasegawa Yat son, Zhen Fumihiko , Alvaro Siza , as well as Le Corbusier , Gaudi, Alvar Aalto , etc. these have been something of a master , the master is to communicate directly with customers and spot performance. So they can run through the whole design in handy when you do the thinking and expression and communication delivery.

\section{Interactive teaching and learning bridges - Design Manuscript}

Students design manuscript musicians like composer, first constructed in the brain , and in the picture or written notes, just a quick recording process on paper only, this process is fully reflects the students of engineering and technical literacy , artistic painting skills a . To become an architect, interior designer must have a good sense of spatial scales , the appliance will be able to learn not to 
use bare hands to the performance of the most creative sense of the painting - the design of the manuscript.

Design is different from painting, teachers design works out for teaching students, students are objects , design something just to express their individuality without considering the student's acceptance and interest only on paper. So , pay attention to the students of communication with customers is the most important, the students should be put through their ideas and demands of artistic expression to be implemented so as to achieve a harmonious design concept resonates teachers and students . Thus the process of teaching the teachers have excellent hand-painted design manuscripts will be subject to the students the ability to respect and welcome.

\section{Construction drawings, renderings efficacy Trio - Design Manuscript}

Students design a manuscript of a good design should be able to both two functions, either as a three-dimensional perspective renderings gives space and immersive experience , but also capable of directing the production of computer graphics staff working drawings on construction workers master can give directly

Currently designers used to express the idea of perspective renderings and construction drawings to guide workers to operate . Draw perspective renderings design intent is a means of birth , in order to trigger the heart of thinking, design gurus often bored ; but I do not know when to begin the separation of art and design drawings , and became two specialized courses and questions, but draw construction plans is tiring work , according to the designer's intent with precise lines painted over molding drawings. We all know that computers can be more accurate renderings are an important means of expression in design manuscript, but at present it is still a chore . Authors believe that the computer is no designer has the passion, the designer is not purely a computer to draw members , should be more use of freehand to capture design inspiration, to emphasize their own ideas, rather than painted works giving a Carpenter gas feelings.

\section{The role of design manuscript}

Design manuscript is present, the effect diagram is at the end, the two can not be reversed. Renderings just a perfect means of performance space, it is unable to leave the design of the manuscript . Design manuscript, which was originally a form of thinking, but it is a manifestation of the spirit of the original , is difficult to replace by any means mother , is among peers, erected between designers and customers a seamless communication link.

\section{Design can release energy - Design manuscripts}

Many people lament creations too little, too serious industry phenomenon of plagiarism collage these are not exaggerated, now in Guangdong, Shanghai and other coastal areas in the indoor and outdoor decoration tender, requires not only look at the computer renderings and design requirements attached to the manuscript as there are reflected in the design of the original force.

\section{" solidified ", promoting the exchange - designed manuscripts}

Potential lies in the design of the manuscript from paper through the eyes to the brain , and then return to the paper 's information circulating among the most peers like to say a napkin story.

This manuscript provides design thinking to visualize the performance of the key design envisages a visual object and the body, so that you can see a lot of information with their peers at the same time, so that it can improve it more deeply , design manuscripts are tentative nature, simple and rough, and the expression is often not comprehensive idea. Precisely because the manuscript reflects the unresolved issues before exploring confused a lot of hard course . Because the manuscript is the carrier of thought, architectural design manuscript can observe various different scenarios, which greatly stimulate people's thinking. Design thinking and design communication should interactions, which implies a new role in the design of the manuscript : the solidification of thinking promotes exchanges, exchanges and exciting three-dimensional thinking ; Reflections supported manuscripts, manuscript but also reflects the thinking, attention focused on the design manuscript in general not a stickler for details . Design manuscript in the form of graphs will manifest manuscript thinking outside the drawing process can be seen as self-talk , in conversation, and the design of the manuscript interaction . Manuscript reproduction design concept, 
its visual image can in turn help and stimulate thinking. You have me, I have you , be good interaction.

\section{Conclusion}

Teaching at University of Art and Design manuscript should be with a distinctive Beaux-Arts , the formation of this style is not one person overnight, but the number of teaching practice in their efforts to draw, the students need to practice pen drawing a solid basic skills and coordination of the brain and hand height. In today 's era of computers widely available, we have to learn hard one new knowledge and skills, not wandering in the traditional techniques, it is necessary to meet the development needs of the times ; Second, there are also discriminating, not to be fooled by the appearance of bewildering skills, computer or, models both mean we demonstrate the design tool only, is a means not an end. Thus, among the teaching reform in colleges and universities today, we still emphasize the importance of art and design of the manuscript, giving it marked emphasis , continue in-depth study of it, and give it a new mission. Students make trained to adapt to the new era of development in line with the times demand the employer, this is the teaching of art and design colleges in the hand-painted with a computer in the true sense.

\section{Acknowledgments}

Xu zhihua (1978-) design of the research direction of the art of Art: Design of art and ceramic art design. Presided over the Jiangxi Provincial Department of culture research project "Jingdezhen traditional culture of the construction industry in the ancient kiln landscape design research", code: YG2014177.

\section{References}

[1] Zhang Fu also edited, foreign craft art history, Beijing : Central Compilation and Translation Press , 1999 .

[2] shuxin Liu,( May) edited by Lee optical power , Bauhaus - the cradle of modern industrial design movement. Beijing: China Light Industry Press , 1988.

[3] Yanzu Li eds., Introduction to Art and Design. Wuhan : Hubei Fine Arts Publishing House , 2002 .

[4] Wang edited by the world of modern architectural history , Beijing : China Building Industry Press, 1999. 